\title{
Semiquantum Expressions for Electronically Nonadiabatic Electron Ejection Rates
}

\author{
Jack Simons \\ Chemistry Department, University of Utah, Salt Lake City, Utah 84112 \\ Received: March 27, 1998
}

\begin{abstract}
Molecular anions that possess excess internal vibrational and/or rotational energy can eject their "extra" electron through a radiationless transition event involving non-Born-Oppenheimer coupling. In such processes, there is an interplay between the nuclear motions (i.e., vibrations and rotations) and the electronic motions that allows energy to be transferred from the former to the latter and that permits momentum and/or angular momentum to also be transferred in a manner that preserves total energy, momentum, and angular momentum. There are well-established quantum mechanical expressions for the state-to-state rates of this kind of radiationless process, and these expressions have been used successfully to compute electron ejection rates. In this paper, we recast the quantum rate equations by making use of approximations that have proven useful in rewriting the quantum expressions for rates of photon absorption in a more classical manner. In so doing, our goal has been to produce rate expressions that allow one to understand, in a more nearly classical manner, the energy-transfer process and to more readily predict when such rates will be significant.
\end{abstract}

\section{Introduction}

A. Relation to Experiments. Numerous series of beautiful specroscopy experiments have been carried out over a number of years in the Lineberger, ${ }^{1}$ Brauman, ${ }^{2}$ and Beauchamp ${ }^{3}$ laboratories in which electronically stable negative molecular ions prepared in excited vibrational-rotational states are observed to eject their "extra" electron. For the anions considered in those experiments, it is unlikely that the anion and neutral-molecule potential energy surfaces undergo crossings at geometries accessed by their vibrational motions in these experiments. It is therefore believed that the mechanism of electron ejection must involve vibration-rotation to electronic energy transfer in which couplings between nuclear motions and electronic motions known as non-Born-Oppenheimer (BO) couplings cause the electron ejection rather than curve crossings in which the anion's energy surface intersects that of the neutral at some geometries.

Certainly, there exist many cases for which the anion and neutral electronic energy curves (surfaces, more generally) intersect. In such cases, the rates of electron ejection are often determined by how frequently the vibrational motion moves the anion into geometries where its energy lies above the neutral's energy (because the rate on electron loss once such a geometry is reached is often ca. $10^{14}-10^{15} \mathrm{~s}^{-1}$ ). This class of electron ejection is not the subject of the present paper; only those anions for which vibration-rotation to electronic energy transfer is the rate-determining event are considered here.

In earlier works, ${ }^{4}$ we and others ${ }^{5}$ have formulated and computed non-BO coupling strengths for several of the anion systems that have been studied experimentally including the following:

1. Dipole-bound anions ${ }^{5 \mathrm{a}, 4 \mathrm{f}}$ in which the extra electron is attracted primarily by the dipole force field of the polar molecule and for which rotation-to-electronic coupling is most important in inducing electron ejection.

2. $\mathrm{NH}^{-}\left(\mathrm{X}^{2} \Pi\right)$ for which ${ }^{4 \mathrm{~d}}$ vibration of the $\mathrm{N}-\mathrm{H}$ bond couples only weakly to the nonbonding $2 \mathrm{p}_{\pi}$ orbital and for which rotation-to-electronic coupling can be dominiant in causing electron ejection for high rotational levels.

3. Enolate anions ${ }^{4 e}$ that have been "heated" by infrared multiple photon absorption for which torsional motion about the $\mathrm{H}_{2} \mathrm{C}-\mathrm{C}$ bond, which destabilizes the $\pi$ orbital containing the extra electron, is the mode contributing most to vibrationto-electronic energy transfer and thus to ejection.

Our calculations have been successful in interpreting trends that are seen in the experimentally observed rates of electron ejection. However, in our opinion, a clear physical picture of the energy, momentum, and angular momentum balancing events that accompany such non-BO processes (i.e., the extra electron must gain energy and change its momentum/angular momentum in a manner opposite to the nuclear motion's loss of energy and change in momentum/angular momentum) has been lacking.

It is the purpose of this paper to enhance our understanding of these events by recasting the rate equations in ways that are less quantum-state specific but that focus on the movements of the nuclei in a nearly classical manner. This is done by

1. starting with the rigorous state-to-state quantum expression for non-BO transition rates, ${ }^{64 \mathrm{~g}}$

2. including what is known from past experience ${ }^{4}$ about the magnitudes and geometry dependencies of the electronic nonBO matrix elements arising in these rate expressions, to

3. make the simplest reasonable approximation to the nuclear motion $^{7}$ (i.e., vibration-rotation) and its coupling to the electronic motions.

By so doing, we are able to arrive at expressions, which we term semiquantum, for rates of electron ejection that, in our opinion, offer better physical insight into these radiationless processes and thus offer the potential for predicting when such rates will be significant in other systems. It should be emphasized that it is not our intent to produce rate equations that will allow more accurate calculations of these electronically nonadiabatic rates. For computing these rates, there is nothing wrong with the fully quantum rate expressions that form the starting point of our treatment. Our approximations to these 
rate expressions, instead, provide expressions that are more readily understood and interpreted than the fully quantum equations.

B. State-to-State Quantum Rate Expression. Within the Born-Oppenheimer approximation, the electronic Schrödinger equation

$$
h_{\mathrm{e}}(r \mid Q) \psi_{k}(r \mid Q)=E_{k}(Q) \psi_{k}(r \mid Q)
$$

is solved to obtain electronic wave functions $\psi_{k}(r \mid Q)$, which are functions of the molecule's electronic coordinates (collectively denoted $r$ ) and atomic coordinates (denoted $Q$ ), and the corresponding electronic energies $E_{k}(Q)$, which are functions of the $Q$ coordinates. The electronic Hamiltonian

$$
\begin{array}{r}
h_{\mathrm{e}}(r \mid Q)=\sum_{i}\left\{-\hbar^{2} / 2 m_{\mathrm{e}} \nabla_{i}^{2}+{ }^{1 / 2} \sum_{j \neq i} e^{2} / r_{i, j}-\sum_{a} Z_{a} e^{2} / r_{i, a}+\right. \\
\left.1 / 2 \sum_{a \neq b} Z_{a} Z_{b} e^{2} / R_{a, b}\right\}
\end{array}
$$

contains, respectively, the sum of the kinetic energies of the electrons, the electron-electron repulsion, the electron-nuclear Coulomb attraction, and the nuclear-nuclear repulsion energy.

Given the solutions to the electronic Schrödinger equation, the solutions of the full Schrödinger equation (i.e., the equation in which all nuclei and electrons are moving)

$$
H(r \mid Q) \Psi(r, Q)=E \Psi(r, Q)
$$

are expressed as sums over the (complete set of functions of the electronic coordinates $r$ ) electronic functions $\left\{\psi_{k}(r \mid Q)\right\}$

$$
\Psi(r, Q)=\sum_{k} \psi_{k}(r \mid Q) \chi_{k}(Q)
$$

with the $\chi_{k}(Q)$ carrying the remaining $Q$ dependence. When substituted into the full Schrödinger

$$
(H-E) \sum_{k} \psi_{k}(r \mid Q) \chi_{k}(Q)=0
$$

then using the fact that the full Hamiltonian $H$ is $h_{\mathrm{e}}$ plus the kinetic energy operator for nuclear motion $T$

$$
H=h_{\mathrm{e}}+T=h_{\mathrm{e}}+\sum_{a}\left(-\hbar^{2} / 2 m_{\mathrm{a}} \nabla_{\mathrm{a}}^{2}\right)
$$

premultiplying the above Schrödinger equation by $\psi_{n}$ and integrating over the electronic coordinates gives the set of coupled equations that need to be solved for the $\left\{\chi_{k}\right\}$ :

$$
\begin{gathered}
\sum_{k} \int \psi_{n}{ }^{*}(r \mid Q)\left\{h_{\mathrm{e}}+T-E\right\} \psi_{k}(r \mid Q) \chi_{k}(Q) \mathrm{d} r= \\
\left\{E_{n}(Q)-E\right\} \chi_{n}(Q)+T \chi_{n}(Q)+ \\
\sum_{a} \sum_{k}\left\{\int \psi_{n}{ }^{*}(r \mid Q)\left(-i \hbar \partial \psi_{k} / \partial R_{a}\right)\left(-i \hbar \partial \chi_{k} / \partial R_{a}\right) / m_{a} \mathrm{~d} r+\right. \\
\left.\int \psi_{n}{ }^{*}(r \mid Q)\left(-\hbar^{2} \partial^{2} \psi_{k} / \partial R_{a}{ }^{2}\right) / 2 m_{a} \mathrm{~d} r \chi_{k}\right\}=0
\end{gathered}
$$

The expression

$$
\left\{E_{n}(Q)-E\right\} \chi_{n}(Q)+T \chi_{n}(Q)=0
$$

is the equation governing the nuclear motion functions $\left\{\chi_{k L^{-}}\right.$ $(Q)\}$ in the absence of the non-Born-Oppenheimer (non-BO) coupling terms (i.e., the latter two terms in eq 7). Within this model, the vibration-rotation functions $\left\{\chi_{k}(Q)\right\}$ of each specific electronic state labeled $k$ are found by solving the vibrationrotation Schrödinger equation

$$
\left\{T+E_{k}(Q)\right\} \chi_{k, L}(Q)=\epsilon_{k, L} \chi_{k, L}(Q)
$$

There are a complete set of functions of $Q$ (i.e., the $\left\{\chi_{k, L}\right\}$ for each electronic state $k$.

In the theory of radiationless transitions as covered in this paper, $6,4 \mathrm{~g}$ the two non-BO terms are treated as perturbations (not externally applied, but arising as imperfections within this model of molecular structure) that can induce transitions between unperturbed states each of which is taken to be a specific BornOppenheimer product state:

$$
\Psi_{k, L}(r, Q) \cong \psi_{k}(r \mid Q) \chi_{k, L}(Q)
$$

It is reasonably well-established that the non-BO coupling term involving second derivatives of the electronic wave function contributes less to the coupling than do the terms $\left(-i \hbar \partial \psi_{k} / \partial R_{a}\right)$ $\left(-i \hbar \partial \chi_{k} / \partial R_{a}\right) / m_{a}$ having first derivatives of the electronic and vibration-rotation functions. Hence, it is only the latter terms that will be discussed further in this paper.

With this background, the rate $R\left(\mathrm{~s}^{-1}\right)$ of transition from a Born-Oppenheimer initial state $\Psi_{\mathrm{i}}=\psi_{\mathrm{i}} \chi_{\mathrm{i}}$ to a final state $\Psi_{\mathrm{f}}$ $=\psi_{\mathrm{f}} \chi_{\mathrm{f}}$ is given, via first-order perturbation theory, as

$R=(2 \pi / \hbar) \int\left|\left\langle\chi_{\mathrm{i}} \mid\left\langle\psi_{\mathrm{i}}|P| \psi_{\mathrm{f}}\right\rangle(P / \mu) \chi_{\mathrm{f}}\right\rangle\right|^{2} \delta\left(\epsilon_{\mathrm{f}}+E-\epsilon_{\mathrm{i}}\right) \rho(E) \mathrm{d} E$

Here, $\epsilon_{\mathrm{i}, \mathrm{f}}$ are the vibration-rotation energies of the initial (anion) and final (neutral) states, and $E$ denotes the kinetic energy carried away by the ejected electron (e.g., the initial state corresponds to an anion and the final state to a neutral molecule plus an ejected electron). The density $\rho$ of translational energy states of the ejected electron is related to the kinetic energy by $\rho(E)=4 \pi m_{\mathrm{e}} L^{3}(2 m e E)^{1 / 2} / \hbar^{2}$. Here and elsewhere, we use the short-hand notation involving $P \psi P \chi / \mu$ to symbolize the action of the multidimensional derivative operators arising in the nonBO couplings

$$
\left(P \psi_{\mathrm{f}}\right)\left(P / \mu \chi_{\mathrm{f}}\right)=\sum_{a}\left(-i \hbar \partial \psi_{\mathrm{f}} / \partial R_{a}\right)\left(-i \hbar \partial \chi_{\mathrm{f}} / \partial R_{a}\right) / m_{a}
$$

where $R_{a}$ runs over the Cartesian coordinates $\left(X_{a}, Y_{a}, Z_{a}\right)$ of the $a$ th atom whose mass is $m_{a}$.

C. The Electronic Non-BO Matrix Elements. The integrals over the anion and neutral-plus-free-electron electronic states

$$
m_{\mathrm{i}, \mathrm{f}}=\left\langle\psi_{\mathrm{f}}|P| \psi_{\mathrm{i}}\right\rangle
$$

are known to be large in magnitude only under special circumstances:

1. The orbital of the anion from which an electron is ejected to form the state $\psi_{\mathrm{f}}$ of the neutral (usually the anion's highest occupied molecular orbital (HOMO)) must be strongly modulated or affected by movement of the molecule in one or more directions $(Q)$. That is, $\partial \psi_{\mathrm{i}} / \partial Q$, which appears in $P \psi_{\mathrm{i}}$, must be significant or the above integral will be small.

2. The state-to-state energy gap $\epsilon_{\mathrm{i}}-\epsilon_{\mathrm{f}}$, which is equal to the energy $E$ of the ejected electron, must not be too large; otherwise, the oscillations in the ejected electron's wave function $\psi_{\mathrm{f}}$ will be so rapid as to render overlap with $\partial \psi_{\mathrm{i}} / \partial Q$ negligible again, making the above integral small.

Moreover, symmetry can cause $m_{\mathrm{i}, \mathrm{f}}=\left\langle\psi_{\mathrm{f}}|P| \psi_{\mathrm{i}}\right\rangle$ to vanish. In particular, if the direct products of the symmetry of $\psi_{\mathrm{i}}$ and 

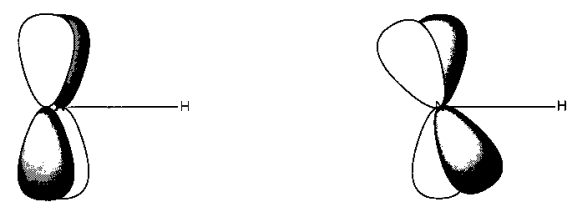

$\mathrm{d} \Psi / \mathrm{dR}=(\Psi(\mathrm{R}+\delta)-\Psi(\mathrm{R})) / \delta$ causes $2 p_{x}$ to acquire $d$ character

$\mathrm{d} \Psi / \mathrm{d} \theta=(\Psi(\theta+\delta)-\Psi(\theta)) / \delta$ causes $2 p_{x}$ to acquire $2 p_{z}$ character

Figure 1. Orbital response of $\mathrm{NH}^{-}$'s $2 \mathrm{p}_{\pi}$ orbital to (a) vibration of the $\mathrm{N}-\mathrm{H}$ bond (left) and (b) rotation of the $\mathrm{N}-\mathrm{H}$ bond (right).

of $\partial / \partial Q$ do not match that of $\psi_{\mathrm{f}}$, then $m_{\mathrm{i}, \mathrm{f}}$ will vanish (i.e., if direct product of the HOMO's symmetry and the symmetry of the vibration or rotation motion from which energy is transferred determines the symmetry of the ejected electron's continuum orbital).

Let us consider these conditions in more detail before proceeding further because they form the basis for approximations that are introduced later and that are important components in our treatment. The derivatives (i.e., the dynamic responses) of the anion's orbitals to nuclear motions $\partial \psi_{\mathrm{i}} / \partial Q$ arise from two sources:

1. The orbital's LCAO-MO coefficients depend on the positions of the atoms (or, equivalently, on the anion's bond lengths and internal angles). For example, the $\pi^{*}$ orbital of an olefin anion that contains the "extra" electron is affected by stretching or twisting the $\mathrm{C}-\mathrm{C}$ bond involving this orbital because the LCAO-MO coefficients depend on the bond length and twist angle. As the bond stretches or twists, the $\pi^{*}$ orbital's LCAO-MO coefficients vary, as a result of which the orbital's energy, radial extent, and other properties also vary.

2. The atomic orbitals (AO) themselves dynamically respond to the motions of the atomic centers. For example, vibration of the $\mathrm{X}^{2} \Pi \mathrm{NH}^{-}$anion's $\mathrm{N}-\mathrm{H}$ bond induces $\mathrm{d}_{\pi}$ character into the $2 \mathrm{p}_{\pi}$ orbital containing the extra electron as shown in Figure 1. Alternatively, rotation of this anion's $\mathrm{N}-\mathrm{H}$ bond axis causes the $2 \mathrm{p}_{\pi}$ HOMO to acquire some $2 \mathrm{p}_{\sigma}$ character (see Figure 1 again). Such AO responses can be evaluated using the same analytical derivative methods that have made computation of potential energy gradients and Hessians powerful tools in quantum chemistry.

Another view of how the LCAO-MO coefficients vary with geometry can be achieved by differentiating $h_{\mathrm{e}} \psi_{\mathrm{i}}=E_{i} \psi_{\mathrm{i}}$ with respect to $Q$ (an arbitrary molecular motion), premultiplying by the anion-plus-free-electron function $\psi_{\mathrm{f}}$, and integrating over the electronic coordinates $r$ to obtain

$$
\left.\left\langle\psi_{\mathrm{f}}\left|\partial h_{\mathrm{e}} / \partial Q\right| \psi_{\mathrm{i}}\right\rangle /\left(E_{\mathrm{i}}-E_{\mathrm{f}}-E\right)=\left\langle\psi_{\mathrm{f}} \mid \partial / \partial Q \psi_{\mathrm{i}}\right\rangle\right\rangle
$$

In this form, one sees that the response of the anion's electronic state, when projected against the neutral-plus-free-electron state to which it will decay, will be enhanced at geometries where the anion and neutral potential surfaces approach closely (so the denominator in eq 13 is small). Enhancement is also effected when the initial and final states have a strong matrix element of the "force operator" $\partial h_{\mathrm{e}} / \partial Q$. The latter is effectively a one-electron operator involving derivatives of the electronnuclear Coulomb attraction potential $\sum_{\mathrm{i}} \sum_{a} Z_{a} e^{2} / r_{\mathrm{ii}, a}$, so the matrix element $\left\langle\psi_{\mathrm{f}}\left|\partial h_{\mathrm{e}} / \partial Q\right| \psi_{\mathrm{i}}\right\rangle$ can be visualized as $\left\langle\phi_{\mathrm{f}}\left|\partial h_{\mathrm{e}} / \partial Q\right| \phi_{\mathrm{i}}\right\rangle$, where $\phi_{\mathrm{i}}$ is the anion's HOMO and $\phi_{\mathrm{f}}$ is the continuum orbital of the ejected electron. At geometries where the anion-neutral energy surfaces are far removed, the denominator in eq 13 will attenuate the coupling. If the state-to-state energy difference $\epsilon_{\mathrm{i}}-\epsilon_{\mathrm{f}}=$ $E$ accompaning the electron ejection is large, the integral $\left\langle\phi_{\mathrm{f}}\right| \partial h_{\mathrm{e}} /$

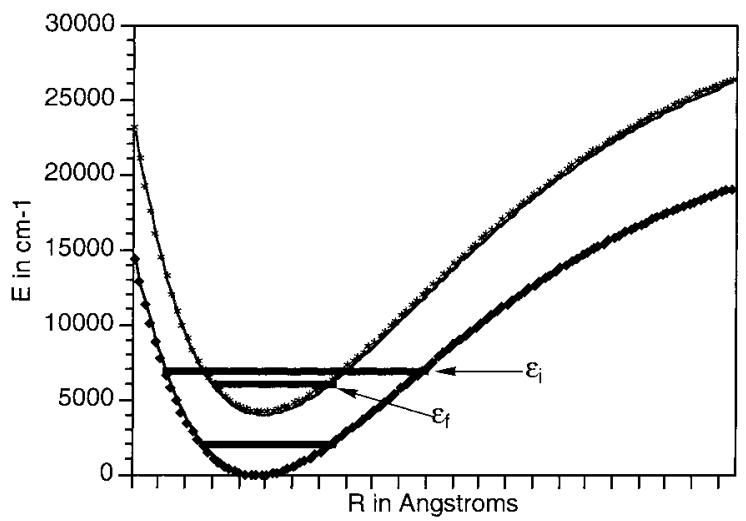

Figure 2. Anion (lower) and neutral (upper) potential energy surfaces illustrative of $\mathrm{NH}^{-}$where the surface spacing does not vary strongly along $R$.

$\partial Q\left|\phi_{\mathrm{i}}\right\rangle$ will be small because the continuum orbital $\phi_{\mathrm{f}}$ will be highly oscillatory and thus will not overlap well with $\left(\partial h_{\mathrm{e}} / \partial Q\right) \phi_{\mathrm{i}}$.

In summary, for non-BO coupling to be significant, ${ }^{4}$ the anion's HOMO must be strongly modulated by a motion (vibration or rotation) of the molecule's nuclear framework and the state-to-state energy gap must not be too large as to render the HOMO-to-continuum-orbital overlap insignificant. For the HOMO to be strongly modulated, it is helpful if the anion and neutral energy surfaces approach closely (n.b., this is not the same as requiring that the state-to-state energy gap $\epsilon_{\mathrm{i}}-\epsilon_{\mathrm{f}}$ be small) at some accessible geometries.

It should be emphasized that it is necessary but not suffficient for $E_{\mathrm{f}}(Q)-E_{\mathrm{i}}(Q)$ to be small over an appreciable range of geometries; this only guarantees that the denominator in eq 13 is small. It is also necessary that $E_{\mathrm{f}}(Q)-E_{\mathrm{i}}(Q)$ decrease at a significant rate as the point of closest approach is reached; this is why we say the surfaces must approach closely. Viewed another way, if $E_{\mathrm{f}}(Q)-E_{\mathrm{i}}(Q)$ were small yet unvarying over some range of geometries $(Q)$, then the HOMO's electron binding energy (and thus radial extent) would remain unchanged over this range of geometries. In such a case, movement along $Q$ would not modulate the HOMO, and thus $\partial \psi_{\mathrm{i}} / \partial Q$ would vanish. Let us consider a few examples to further illustrate.

D. A Few Examples. In Figure 2 are depicted anion and neutral potential curves that are qualitatively illustrative of ${ }^{1 b, 4 d}$ the $\mathrm{X}^{2} \Pi \mathrm{NH}^{-}$case mentioned earlier. In this anion, the HOMO is a nonbonding $2 \mathrm{p}_{\pi}$ orbital localized almost entirely on the $\mathrm{N}$ atom. As such, its LCAO-MO coefficients are not strongly affected by vibration of the $\mathrm{N}-\mathrm{H}$ bond (because it is a nonbonding orbital). Moreover, the anion and neutral surfaces have nearly identical $R_{\mathrm{e}}$ and $\omega_{\mathrm{e}}$ values, and similar $D_{\mathrm{e}}$ values, as a result of which these two surfaces are nearly parallel to one another over a wide range of internuclear distances and are separated by ca. $0.4 \mathrm{eV}$ or more than $3000 \mathrm{~cm}^{-1}$ at their minima. It has been seen experimentally that excitation of $\mathrm{NH}^{-}$to the low rotational states of the $v=1$ vibrational level (which lies above $v=0 \mathrm{NH}$ of the neutral and thus has enough energy to eject the electron) results in very slow (e.g., ca. $10^{8} \mathrm{~s}^{-1}$ ) electron ejection, corresponding to ca. 1 million vibrational periods before detachment occurs. However, excitation to high rotational levels (e.g., $J=40$ ) of $v=1$ produces much more rapid electron ejection (ca. $10^{9}-10^{10} \mathrm{~s}^{-1}$ ). These data have been interpreted as saying that vibrational coupling is weak (i.e., $\partial \psi_{\mathrm{i}} /$ $\partial R$ is small) because of the nonbonding nature of the $2 \mathrm{p}_{\pi} \mathrm{MO}$, while rotational coupling becomes significant (i.e., $\partial \psi_{\mathrm{i}} / \partial \theta$ is large) for high $J$.

In Figure 3 are shown anion and neutral potential curves, as functions of the "twist" angle of the $\mathrm{H}_{2} \mathrm{C}-\mathrm{C}$ bond in a typical 


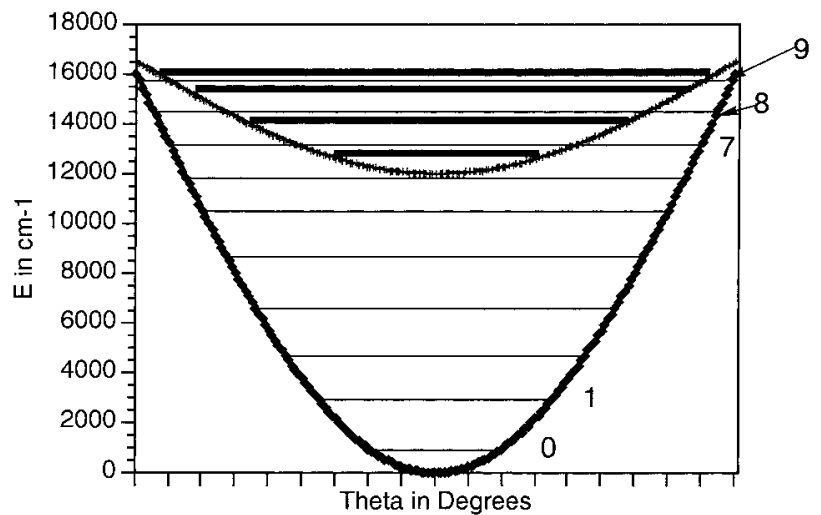

Figure 3. Anion (lower) and neutral (upper) potential energy surfaces illustrative of enolate cases where the surface spacing varies strongly along the $\mathrm{H}_{2} \mathrm{C}-\mathrm{C}$ torsion angle $\theta$ and becomes very small near $\theta=$ $90^{\circ}$.

enolate anion ${ }^{2,4 e}$ such as acetaldehyde enolate $\mathrm{H}_{2} \mathrm{CCHO}^{-}$. Angles near $\theta=0$ correspond to geometries where the $\mathrm{p}_{\pi}$ orbital of the $\mathrm{H}_{2} \mathrm{C}$ moiety is delocalized over the two $\mathrm{p}_{\pi}$ orbitals of the neighboring $\mathrm{C}$ and $\mathrm{O}$ atoms, thus forming a delocalized $\pi$ HOMO. At angles near $\theta=90^{\circ}$, the $\mathrm{p}_{\pi}$ orbital of the $\mathrm{H}_{2} \mathrm{C}$ group is no longer stabilizated by delocalization, so the HOMO's energy is much higher (as a result of which the anion-neutral surfaces approach closely). In this case, excitation of, for example, $v=7$ in the $\mathrm{H}_{2} \mathrm{C}-\mathrm{C}$ torsional mode of the anion might be expected to produce electron ejection because $v=7$ of the anion lies above $v=0$ of the neutral. However, over the range of $\theta$ values accessible to both the $v=7$ vibrational function of the anion and the $v=0$ function of the neutral, the anionneutral energy-surface gap is quite large (i.e., $E_{\mathrm{f}}(Q)-E_{\mathrm{i}}(Q)$ is large even though $\epsilon_{\mathrm{i}}-\epsilon_{\mathrm{f}}$ is small). In contrast, excitation of $v$ $=9$ of the anion could produce more rapid electron ejection (to $v=2$ of the neutral, but not to $v=0$ of the neutral) because for the $v=9 \rightarrow v=2$ transition there are angles accessed by both $v=9$ anion and $v=2$ neutral vibrational functions for which $E_{\mathrm{f}}(Q)-E_{\mathrm{i}}(Q)$ is small and changing; moreover, the stateto-state gap $\epsilon_{\mathrm{i}}-\epsilon_{\mathrm{f}}$ is also small in this case.

The purpose of these examples and of considering the nature of the electronic non-BO matrix element was to prepare for critical approximations that are to be introduced. In particular

1. we will focus on transitions for which $\epsilon_{\mathrm{i}}-\epsilon_{\mathrm{f}}$ is small;

2. we will focus on molecular deformations that most strongly modulate the anion's HOMO, so

3 . we will focus on geometries $Q$ near which the anionneutral surface spacing is small and changing.

\section{Time Correlation Function Expression for Rates}

Before dealing further with the non-Born-Oppenheimer case, it is useful to recall how one can cast other rate expressions, such as the rate of photon absorption ${ }^{7}$ accompanying an electronic transition in a molecule, in terms of a Fourier transform of a time-dependent function that involves dynamical motions on the initial and final electronic states' potential energy surfaces.

A. The Optical Spectroscopy Case. 1. From WentzelFermi Golden Rule to the Time Domain. The expression for the rate $R\left(\mathrm{~s}^{-1}\right)$ of photon absorption due to coupling $V$ between a molecule's electronic and nuclear charges and an electromagnetic field is given through first order in perturbation theory by the well-known Wentzel-Fermi "golden rule: formula: 7,8

$$
R=(2 \pi / \hbar)\left|\left\langle\psi_{\mathrm{i}} \chi_{\mathrm{i}}|V| \psi_{\mathrm{f}} \chi_{\mathrm{f}}\right\rangle\right|^{2} \delta\left(\epsilon_{\mathrm{f}}-\epsilon_{\mathrm{i}}-\hbar \omega\right)
$$

Here, $\psi_{\mathrm{i}, \mathrm{f}}$ and $\chi_{\mathrm{i}, \mathrm{f}}$ are the initial and final state electronic and vibration-rotation state wave functions, respectively, and $\epsilon_{\mathrm{i}, \mathrm{f}}$ are the respective state energies, which are connected via a photon of energy $\hbar \omega$. For a particular electronic transition (i.e., a specific choice for $\psi_{\mathrm{i}}$ and $\psi_{\mathrm{f}}$ and for a specific choice of initial vibration-rotation state), it is possible to obtain an expression for the total rate $R_{\mathrm{T}}$ of transitions from this particular initial state into all vibration-rotation states of the final electronic state. This is done by using the Fourier representation of the Dirac $\delta$ function

$$
\delta\left(\epsilon_{\mathrm{f}}-\epsilon_{\mathrm{i}}-\hbar \omega\right)=(1 / 2 \pi \hbar) \int \exp \left[i t\left(\epsilon_{\mathrm{f}}-\epsilon_{\mathrm{i}}-\hbar \omega\right) / \hbar\right] \mathrm{d} t
$$

and summing over the indices labelling the final vibrationrotation states $\chi_{\mathrm{f}}$

$$
\begin{aligned}
& R_{\mathrm{T}}=(2 \pi / \hbar) \sum_{f}(1 / 2 \pi \hbar) \int \exp \left[i t\left(\epsilon_{\mathrm{f}}-\epsilon_{\mathrm{i}}-\hbar \omega\right) / \hbar\right] \times \\
&\left\langle\psi_{\mathrm{i}} \chi_{\mathrm{i}}|V| \psi_{\mathrm{f}} \chi_{\mathrm{f}}\right\rangle\left\langle\psi_{\mathrm{i}} \chi_{\mathrm{i}}|V| \psi_{\mathrm{f}} \chi_{\mathrm{f}}\right\rangle^{*} \mathrm{~d} t
\end{aligned}
$$

Next, one introduces the electronic transition matrix element (which may be the electric dipole matrix element, but need not be so restricted for the delelopment presented here)

$$
\mu_{\mathrm{i}, \mathrm{f}}=\left\langle\psi_{\mathrm{f}}|V| \psi_{\mathrm{i}}\right\rangle
$$

and uses the facts that the $\psi_{\mathrm{i}, \mathrm{f}}$ are eigenfuncions of the electronic Hamiltonian $h_{\mathrm{e}}$ and that the $\chi_{\mathrm{i}, \mathrm{f}}$ are eigenfuntions of the respective vibration-rotation Hamiltonia $T+V_{\mathrm{i}, \mathrm{f}}(Q)$ belonging to the two electronic states having potential energies $V_{\mathrm{i}, \mathrm{f}}(Q)$ and vibration-rotation kinetic energy $T$ (both of which are functions of the molecule's atomic position coordinates collectively denoted $Q$ )

$$
h_{\mathrm{e}} \psi_{\mathrm{i}, \mathrm{f}}=V_{\mathrm{i}, \mathrm{f}}(Q) \psi_{\mathrm{i}, \mathrm{f}} \quad \text { and } \quad\left[T+V_{\mathrm{i}, \mathrm{f}}(Q)\right] \chi_{\mathrm{i}, \mathrm{f}}=\epsilon_{\mathrm{i}, \mathrm{f}} \chi_{\mathrm{i}, \mathrm{f}}
$$

These identities then allow $R_{\mathrm{T}}$ to be rewritten as

$$
\begin{aligned}
& R_{\mathrm{T}}= \\
& \begin{aligned}
(2 \pi / \hbar) \sum_{\mathrm{f}}(1 / 2 \pi \hbar) \int \exp [-i t \omega] & \left\langle\chi_{\mathrm{i}}\left|\exp \left(-i t h_{\mathrm{i}} / \hbar\right) \mu_{\mathrm{i}, \mathrm{f}} *\right| \chi_{\mathrm{f}}\right\rangle \times \\
& \left\langle\chi_{\mathrm{f}} \exp \left(i t h_{\mathrm{f}} / \hbar\right)\left|\mu_{\mathrm{i}, \mathrm{f}}\right| \chi_{\mathrm{i}}\right\rangle \mathrm{d} t
\end{aligned}
\end{aligned}
$$

In this form, the completeness of the $\left\{\chi_{\mathrm{f}}\right\}$

$$
\sum_{\mathrm{f}}\left|\chi_{\mathrm{f}}\right\rangle\left\langle\chi_{\mathrm{f}}\right|=1
$$

can be used to eliminate the sum over the vibration-rotation states belonging to the final electronic state and thus express $R_{\mathrm{T}}$ in the following manner:

$$
\begin{array}{r}
R_{\mathrm{T}}=(2 \pi / \hbar)(1 / 2 \pi \hbar) \int \exp [-i t \omega]\left\langle\exp \left(i t h_{\mathrm{i}} / \hbar\right) \chi_{\mathrm{i}}\right| \mu_{\mathrm{i}, \mathrm{f}} * \times \\
\exp \left(i t h_{\mathrm{f}} / \hbar\right) \mu_{\mathrm{i}, \mathrm{f}}\left|\chi_{\mathrm{i}}\right\rangle \mathrm{d} t
\end{array}
$$

The above expression is often visualized (and computed) ${ }^{9}$ in terms of the Fourier transform of the overlap of two timepropagated wave fuctions:

(a) One function $F_{1}$ is equal to the initial vibration-rotation function $\left|\chi_{i}\right\rangle$ upon which the electronic transition perturbation $\mu_{\mathrm{i}, \mathrm{f}}$ acts, after which the resultant product function is propagated for a time $t$ on the final-state's potential energy surface by using the propagator $\exp \left(i t h_{\mathrm{f}} / \hbar\right)$. 
(b) The second function $F_{2}$ is equal to the initial vibrationrotation function $\left|\chi_{\mathrm{i}}\right\rangle$, which is propagated for time $t$ on the initial-state's potential energy surface using the propagator exp$\left(i t h_{\mathrm{i}} / \hbar\right)$ (which, of course simpy produces $\left.\exp \left(i t \epsilon_{\mathrm{i}} / \hbar\right) \times\left|\chi_{\mathrm{i}}\right\rangle\right)$, after which the electronic transition perturbation $\mu_{\mathrm{i}, \mathrm{f}}$ is allowed to act.

The overlap $\left\langle F_{2} \mid F_{1}\right\rangle$ is then Fourier transformed at the energy $\hbar \omega$ of the photon to obtain the rate $R_{\mathrm{T}}$ of absorption of photons of frequency $\omega$.

Before returning to the non-BO rate expression, it is important to note that, in this electronic-transition spectroscopy case, the perturbation (i.e., the photon's electromagnetic potential) appears explicitly only in the electronic $\mu_{\mathrm{i}, \mathrm{f}}$ matrix element because this external field is purely an electronic operator. In contrast, in the non-BO case, the perturbation involves a product of momentum operators, one acting on the electronic wave function $\left(P \psi_{\mathrm{i}}\right)$ the second $\left(P \psi_{\mathrm{i}} / \mu\right)$ acting on the vibration/rotation wave function because the non-BO perturbation involves an explicit exchange of energy and momentum between the electrons and the nuclei rather than an absorption of energy and momentum from a photon. As a result, one has matrix elements of the form $\left\langle\chi_{\mathrm{i}} \mid\left\langle\psi_{\mathrm{i}}|P| \psi_{\mathrm{f}}\right\rangle(P / \mu) \chi_{\mathrm{f}}\right\rangle$ in the non-BO case whereas one finds $\left\langle\chi_{\mathrm{i}}\left|\left\langle\psi_{\mathrm{i}}|V| \psi_{\mathrm{f}}\right\rangle\right| \chi_{\mathrm{f}}\right\rangle$ in the spectroscopy case. A primary difference is that derivatives of the vibration/rotation functions appear in the former case (in $(P / \mu) \chi)$ while only $\chi$ appears in the latter.

2. The Semiclassical Approximation to $R_{T}$. The most classical (and hence, least quantum) approximation ${ }^{7}$ is to ignore the fact that the nuclear-motion kinetic energy operator $T$ does not commute with the potentials $V_{\mathrm{i}, \mathrm{f}}$ and thus to write

$$
\exp \left(i t h_{\mathrm{i}, \mathrm{f}} / \hbar\right) \cong \exp (i t T / \hbar) \exp \left(i t V_{\mathrm{i}, \mathrm{f}} / \hbar\right)
$$

Inserting this into the above equation for $R_{\mathrm{T}}$, and also assuming that $T$ also commutes with $\mu_{\mathrm{i}, \mathrm{f}}$ (or that $\mu_{\mathrm{i}, \mathrm{f}}$ does not depend significantly on geometry $Q$ ), gives

$$
\begin{array}{r}
R_{\mathrm{T}}=(2 \pi / \hbar)(1 / 2 \pi \hbar) \int \exp [-i t \omega]\left\langle\chi_{\mathrm{i}} \exp \left(i t V_{\mathrm{i}} / \hbar\right)\right| \mu_{\mathrm{i}, \mathrm{f}}{ }^{*} \times \\
\left.\exp \left(i t V_{\mathrm{f}} / \hbar\right)\left|\mu_{\mathrm{i}, \mathrm{f}}\right| \chi_{\mathrm{i}}\right\rangle \mathrm{d} t
\end{array}
$$

This treatment amounts to treating the nuclear motions fully classically while using quantum mechanics for the electronic motions. Carrying out the Fourier integral over time in eq 23 gives

$$
(1 / 2 \pi \hbar) \int \exp \left[i t\left(V_{\mathrm{f}}-V_{\mathrm{i}}-\hbar \omega\right) / \hbar\right] \mathrm{d} t=\delta\left(V_{\mathrm{f}}-V_{\mathrm{i}}-\hbar \omega\right)
$$

This $\delta$ function can be used in the expression for $R_{\mathrm{T}}$ to constrain the multidimensional integral over vibration-rotation coordinates (denoted $Q$ ) to those specific values that obey the energy conservation condition

$$
\hbar \omega=V_{\mathrm{f}}(Q)-V_{\mathrm{i}}(Q)
$$

thereby yielding

$$
R_{\mathrm{T}}=(2 \pi / \hbar)\left\langle\chi_{\mathrm{i}}\left|\mu_{\mathrm{i}, \mathrm{f}} * \delta\left(V_{\mathrm{f}}-V_{\mathrm{i}}-\hbar \omega\right) \mu_{\mathrm{i}, \mathrm{f}}\right| \chi_{\mathrm{i}}\right\rangle
$$

This semiclassical (quantum electrons and classical nuclear motions) result can be interpreted as saying that $R_{\mathrm{T}}$ is given as the norm of the function $\mu_{\mathrm{i}, \mathrm{f}} \chi_{\mathrm{i}}$ (consisting of the perturbation $\mu_{\mathrm{i}, \mathrm{f}}$ acting on the initial vibration-rotation state) constrained to those regions of space that obey the condition $\hbar \omega=V_{\mathrm{f}}(Q)$ - $V_{\mathrm{i}}(Q)$. This condition is equivalent to constraining the integration to those regions where the change in classical

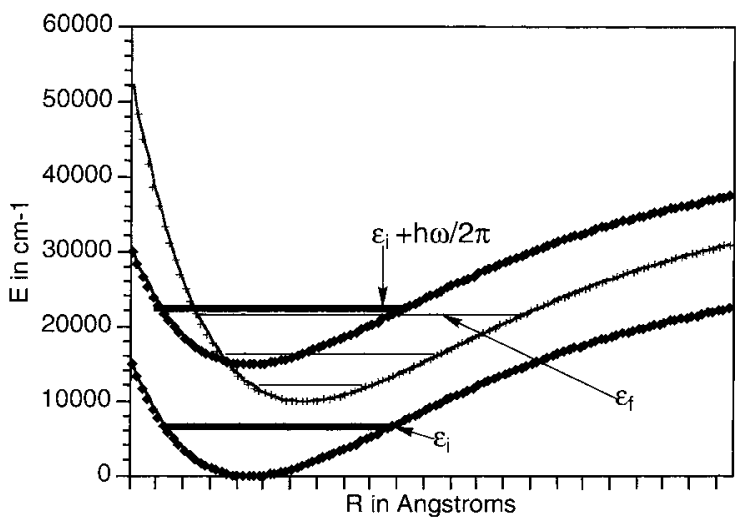

Figure 4. Anion (lowest) and neutral (second lowest at large $R$ ) potential energy surfaces arising in the photon absorption case. The curve that is highest in energy at large $R$ is the anion surface that has been shifted upward in energy by the photon's energy $\hbar \omega$ (which has been chosen to make $\epsilon_{\mathrm{f}}$ equal to $\epsilon_{\mathrm{i}}$ plus the photon's energy).

nuclear-motion kinetic energy in moving from the initial-state surface $V_{\mathrm{i}}$ to the final-state surface $V_{\mathrm{f}}$ is zero. One can visualize such geometies as those at which the upper potential energy surface $V_{\mathrm{f}}(Q)$ is intersected by the lower surface $V_{\mathrm{i}}(Q)$ once the lower surface is shifted to higher energy by an amount $\hbar \omega$. In Figure 4 is shown such an intersection between a $V_{\mathrm{f}}(Q)$ surface and a lower-state $V_{\mathrm{i}}(Q)$ surface that has been shifted upward in energy. In this example, transitions between the initial level $\epsilon_{\mathrm{i}}$ and the final level $\epsilon_{\mathrm{f}}$, whose difference $\epsilon_{\mathrm{f}}-\epsilon_{\mathrm{i}}=$ $\hbar \omega$ determines the energy shift, would occur at $R$ values near where the two surfaces cross.

B. The Non-Born-Oppenheimer Case. 1. From Wentzel-Fermi Golden Rule to the Time Domain. Let us now consider how similar the expression for rates of radiationless transitions induced by non-Born-Oppenheimer couplings can be made to the expressions given above for photon absorption rates. We begin with the corresponding 6 ,4g Wentzel-Fermi "golden rule" expression given in eq 10 for the transition rate between electronic states $\psi_{\mathrm{i}, \mathrm{f}}$ and corresponding vibrationrotation states $\chi_{\mathrm{i}, \mathrm{f}}$ appropriate to the non-BO case:

$$
R=(2 \pi / \hbar) \int\left|\left\langle\chi_{\mathrm{i}} \mid\left\langle\psi_{\mathrm{i}}|P| \psi_{\mathrm{f}}\right\rangle(P / \mu) \chi_{\mathrm{f}}\right\rangle\right|^{2} \delta\left(\epsilon_{\mathrm{f}}+E-\epsilon_{\mathrm{i}}\right) \rho(E) \mathrm{d} E
$$

We recall that $\epsilon_{i, f}$ are the vibration-rotation energies of the molecule in the anion and neutral molecule states, $E$ denotes the kinetic energy carried away by the ejected electron, and the density of translational energy states of the ejected electron is $\rho(E)$. Also recall that we use the shorthand notation to symbolize the multidimensional derivative operators that arise in non-BO couplings and that embody the momentum exchange between the vibration/rotation and electronic degrees of freedom

$$
\left(P \psi_{\mathrm{f}}\right)\left(P / \mu \chi_{\mathrm{f}}\right)=\sum_{a}\left(-i \hbar \partial \psi_{\mathrm{f}} / \partial R_{\mathrm{a}}\right)\left(-i \hbar \partial \chi_{\mathrm{f}} / \partial R_{a}\right) / m_{a}
$$

where $R_{a}$ is one of the Cartesian coordinates $\left(X_{a}, Y_{a}, Z_{a}\right)$ of the $a$ th atom whose mass is $m_{a}$.

In the event that some subset $\left\{Q_{\mathrm{i}}\right\}$ of internal vibration or rotation coordinates has been identified as inducing the radiationless transition, $\left(P \psi_{\mathrm{f}}\right)\left(P / \mu \chi_{\mathrm{f}}\right)$ would represent $\sum_{j}\left(-i \hbar \partial \psi_{\mathrm{f}} / \partial Q_{j}\right)$ $\left(-i \hbar \partial \chi_{\mathrm{f}} / \partial Q_{j}\right) /\left(\mu_{j}\right)$, where $\mu_{\varphi}$ is the reduced mass associated with the coordinate $Q_{j}$. As indicated in the discussion of Section I.C, it is usually straightforward to identify which distortional modes need to be considered by noting which modes most strongly modulate the anion's HOMO. So, for the remainder 
of this work, we will assume that such active modes have been identified as a result of which the sum $\sum_{j}\left(-i \hbar \partial \psi_{\mathrm{f}} / \partial Q_{j}\right)\left(-i \hbar \partial \chi_{\mathrm{f}} /\right.$ $\left.\partial Q_{j}\right) /\left(\mu_{j}\right)$ will include only these modes. The integration over all of the other coordinates contained in the matrix element $\left\langle\left.\chi_{\mathrm{i}}\right|^{-}\right.$ $\left.\left\langle\psi_{\mathrm{i}}|P| \psi_{\mathrm{f}}\right\rangle(P / \mu) \chi_{\mathrm{f}}\right\rangle$ in eq 10 can then be carried out (assuming the electronic element $\left\langle\psi_{\mathrm{i}}|P| \psi_{\mathrm{f}}\right\rangle$ to not depend significantly on these coordinates) to produce an effective Franck-Condon-like factor (FC):

$$
\begin{gathered}
\left\langle\chi_{\mathrm{i}} \mid\left\langle\psi_{\mathrm{i}}|P| \psi_{\mathrm{f}}\right\rangle(P / \mu) \chi_{\mathrm{f}}\right\rangle \\
=\prod_{j=\text { inactive }} \int \mathrm{d} Q_{j}\left\langle\chi_{i, j} \mid \chi_{\mathrm{f}, j}\right\rangle \prod_{j=\text { active }} \int \mathrm{d} Q_{j}\left\langle\chi_{\mathrm{i}, j}\right|\left\langle\psi_{\mathrm{i}}|P| \psi_{\mathrm{f}}\right\rangle \times \\
=\operatorname{FC~} \prod_{j=\text { active }} \int \mathrm{d} Q_{j}\left\langle\chi_{\mathrm{i}, j} \mid\left\langle\psi_{\mathrm{i}}|P| \psi_{\mathrm{f}}\right\rangle(\mathrm{P} / \mu) \chi_{\mathrm{f}, j}\right\rangle
\end{gathered}
$$

Since, by assumption, the anion and neutral molecule do not differ significantly in their geometries (and vibrational frequencies) along the coordinates contributing to the FC factor (otherwise, the anion-neutral energy gap would depend substantially on these modes), the FC factor is probably close to unity in magnitude. Hence, for the remainder of this paper, we will focus only on the active-mode part of this expression and will do so assuming only one such mode is operative (i.e., we treat one active mode at a time).

Introducing the electronic coupling matrix element

$$
m_{\mathrm{i}, \mathrm{f}}=\left\langle\psi_{\mathrm{f}}|P| \psi_{\mathrm{i}}\right\rangle
$$

which plays a role analogous to the $\mu_{\mathrm{i}, \mathrm{f}}$ of photon absorption theory, and realizing that $P$ is a Hermitian operator, allows the non-BO rate $R$ to be rewritten as

$$
\begin{array}{r}
R=(2 \pi / \hbar) \int\left\langle(P / \mu) \chi_{\mathrm{i}}\left|m_{\mathrm{i}, \mathrm{f}} *\right| \chi_{\mathrm{f}}\right\rangle\left\langle\chi_{\mathrm{f}} \mid m_{\mathrm{i}, \mathrm{f}}(P / \mu) \chi_{\mathrm{i}}\right\rangle \delta\left(\epsilon_{\mathrm{f}}+E-\right. \\
\left.\epsilon_{\mathrm{i}}\right) \rho(E) \mathrm{d} E
\end{array}
$$

If the Fourier integral representation of the $\delta$ function is introduced and the sum over all possible final-state vibrationrotation states $\left\{\chi_{\mathrm{f}}\right\}$ is carried out, the total rate $R_{\mathrm{T}}$ appropriate to this non-BO case can be expressed as

$$
\begin{aligned}
R_{\mathrm{T}}=(2 \pi / \hbar) & \sum_{\mathrm{f}} \int(1 / 2 \pi \hbar) \int \exp \left[i t\left(\epsilon_{\mathrm{f}}-\epsilon_{\mathrm{i}}+E\right) / \hbar\right] \times \\
& \left\langle(P / \mu) \chi_{\mathrm{i}}\left|m_{\mathrm{i}, \mathrm{f}} *\right| \chi_{\mathrm{f}}\right\rangle\left\langle\chi_{\mathrm{f}} \mid m_{\mathrm{i}, \mathrm{f}}(P / \mu) \chi_{\mathrm{i}}\right\rangle \mathrm{d} t \rho(E) \mathrm{d} E
\end{aligned}
$$

The next step is to replace $\left(\epsilon_{\mathrm{f}}+E\right)\left\langle\chi_{\mathrm{f}}\right|$ by $\left\langle\chi_{\mathrm{f}}\right|\left(T+V_{\mathrm{f}}+E\right)$ and $\left(\epsilon_{\mathrm{i}}\right)\left|\chi_{\mathrm{i}}\right\rangle$ by $\left|\left(T+V_{\mathrm{i}}\right) \chi_{\mathrm{i}}\right\rangle$ and to use $\sum_{\mathrm{f}}\left|\chi_{\mathrm{f}}\right\rangle\left\langle\chi_{\mathrm{f}}\right|=1$, which gives

$$
\begin{aligned}
R_{\mathrm{T}}=(2 \pi / \hbar) \int(1 / 2 \pi \hbar) \int \rho(E)\left\langle m_{\mathrm{i}, \mathrm{f}}(P / \mu) \exp (i t(T+\right. \\
\left.\left.V_{\mathrm{i}}\right) \hbar\right) \chi_{\mathrm{i}}\left|\exp \left(i t\left(E+T+V_{\mathrm{f}}\right) / \hbar\right) m_{\mathrm{i}, \mathrm{f}}(P / \mu) \chi_{\mathrm{i}}\right\rangle \mathrm{d} t \mathrm{~d} E
\end{aligned}
$$

In this form, the rate expression looks much like that given for the photon absorption rate in eq 21 but with $m_{\mathrm{i}, \mathrm{f}}(P / \mu)$ replacing the molecule-photon transition matrix element $\mu_{\mathrm{i}, \mathrm{f}}$. As in the absorption case, one can view (and even compute) $R_{\mathrm{T}}$ as the Fourier transform of the overlap of two time-propagated functions:

(a) The first $F_{1}$ is the initial vibration-rotation state $\chi_{i}$ upon which the non-BO perturbation $m_{\mathrm{i}, \mathrm{f}}(P / \mu)$ acts after which propagation on the neutral molecule's potential surface $V_{\mathrm{f}}$ is effected via $\exp \left(i t\left(T+V_{\mathrm{f}}\right) / \hbar\right)$.

(b) The second $F_{2}$ is the initial function $\chi_{\mathrm{i}}$ which is propagated on the anion's surface $V_{\mathrm{i}}$ via $\exp \left(i t\left(T+V_{\mathrm{i}}\right) / \hbar\right)$ (producing, of course, $\left.\exp \left(i t \epsilon_{\mathrm{i}} / \hbar\right) \chi_{\mathrm{i}}\right)$ after which the perturbation $m_{\mathrm{i}, \mathrm{f}}(P / \mu)$ is allowed to act.

The time-dependent overlap $\left\langle F_{2} \mid F_{1}\right\rangle$ is then Fourier transformed at energy $E=\epsilon_{\mathrm{i}}-\epsilon_{\mathrm{f}}$ and multiplied by the density of states $\rho(E)$ appropriate to the electron ejected with kinetic energy E.

It should be noted that to use the above time-domain formulas for computing rates, one would need an efficient means of propagating wave packets on the neutral and anion surfaces that would be valid for longer times than are needed in the optical spectroscopy case. Why? Because, in the non-BO situation, the $\left\langle F_{2} \mid F_{1}\right\rangle$ product is multiplied by $\exp (i E t / \hbar)$ and then integrated over time. In the spectroscopy case, $\left\langle F_{2} \mid F_{1}\right\rangle$ is multiplied by $\exp (-i \omega t)$ and integrated over time. In the former case, $E$ corresponds to a small energy difference $E=\epsilon_{\mathrm{i}}-\epsilon_{\mathrm{f}}$, whereas $\hbar \omega$ is equal to the energy of the optical spectroscopic transition. Hence, short-time propagators give sufficiently accurate $\left\langle F_{2} \mid F_{1}\right\rangle$ functions to use in the spectroscopy case, but longer time propagations will be needed in the non-BO case.

2. The Simplest Semiclassical Approximation Fails in the Non-BO Case. If one attempts to follow the photon absorption derivation and make the assumption that the nuclear-motion kinetic energy operator $T$ commutes with $V_{\mathrm{i}, \mathrm{f}}$ and with $m_{\mathrm{i}, \mathrm{f}}$ (n.b., $T$ does commute with $P(\mu)$, the following expression is obtained for $R_{\mathrm{T}}$ :

$$
\begin{gathered}
R_{\mathrm{T}}=(2 \pi / \hbar) \int(1 / 2 \pi \hbar) \int \rho(E) \\
\left\langle m_{\mathrm{i}, \mathrm{f}}(P / \mu) \exp \left(i t\left(V_{\mathrm{i}}\right) \hbar\right) \chi_{\mathrm{i}} \mid \exp \left(i t\left(E+V_{\mathrm{f}}\right) \hbar\right) m_{\mathrm{i}, \mathrm{f}}(P / \mu) \chi_{\mathrm{i}}\right\rangle \mathrm{d} t \mathrm{~d} E
\end{gathered}
$$

The Fourier integral over time can be carried out, and one obtains

$R_{\mathrm{T}}=(2 \pi / \hbar) \int \rho(E)\left\langle m_{\mathrm{i}, \mathrm{f}}(P / \mu) \chi_{\mathrm{i}}\right| \delta\left(V_{\mathrm{f}}+E-V_{\mathrm{i}}\right) m_{\mathrm{i}, \mathrm{f}}$

$$
\left.(P / \mu) \chi_{\mathrm{i}}\right\rangle \mathrm{d} E
$$

For anions that are electronically bound, the anion's electronic energy $V_{\mathrm{i}}(Q)$ lies below the neurtral molecule's electronic energy $V_{\mathrm{f}}(Q)$ as depicted in Figures 2-4. Hence, because $E$ is a positive quantity, there are no geometries for which the argument of the $\delta$ function in the above expression vanishes and, as a result, the non-BO rate cannot be cast in terms of shifted intersecting energy surfaces as can the photon absorption rate.

Therefore, the simplest classical treatment in which the propagator $\exp (i t(T+V) \hbar)$ is approximated in the product form $\exp (i t(T) / \hbar) \exp (i t(V) / \hbar)$ and the nuclear kinetic energy $T$ is conserved during the "transition" produces a nonsensical approximation to the non-BO rate. This should not be surprising because (a) in the photon absorption case, the photon induces a transition in the electronic degrees of freedom that subsequently cause changes in the vibration-rotation energy, while (b) in the non-BO case, the electronic and vibration-rotation degrees of freedom must simultaneously interchange energy and momentum and/or angular momentum, which is impossible to do without the nuclei recoiling.

3. A Semiquantum Approximation That Works. The matrix element occuring in the non-BO rate equation

$$
\begin{array}{r}
M=\left\langle m_{\mathrm{i}, \mathrm{f}}(P / \mu) \exp \left(i t\left(T+V_{\mathrm{i}}\right) / \hbar\right) \chi_{\mathrm{i}}\right| \exp (i t(E+T+ \\
\left.\left.\left.V_{\mathrm{f}}\right) / \hbar\right) m_{\mathrm{i}, \mathrm{f}}(P / \mu) \chi_{\mathrm{i}}\right\rangle
\end{array}
$$

can more fruitfully be handled by 
(a) replacing $\exp \left(i t\left(T+V_{\mathrm{i}}\right) / \hbar\right) \chi_{\mathrm{i}}$ by the exact quantum equivalent $\exp \left(i t\left(\epsilon_{\mathrm{i}}\right) \hbar\right) \chi_{\mathrm{i}}$ and

(b) approximating $\exp \left(i t\left(E+T+V_{\mathrm{f}}\right) / \hbar\right)$ by $\exp (i t(E+T) /$ $\hbar) \exp \left(i t\left(V_{\mathrm{f}}\right) / \hbar\right)$, thus achieving

$$
\begin{aligned}
M=\left\langle m_{\mathrm{i}, \mathrm{f}}(P / \mu) \exp \left(i t\left(\epsilon_{\mathrm{i}}\right) / \hbar\right) \chi_{\mathrm{i}}\right| & \exp (i t(E+T) / \hbar) \times \\
& \exp \left(i t\left(V_{\mathrm{f}}\right) m_{\mathrm{i}, \mathrm{f}}(P / \mu) \chi_{\mathrm{i}}\right\rangle
\end{aligned}
$$

Now introducing completeness relations in the forms $\int \mathrm{d} p|p\rangle$ $\langle p|=1$ and $\int \mathrm{d} Q|Q\rangle\langle Q|=1$ and using $\left(T+V_{\mathrm{i}}\right) \chi_{\mathrm{i}}=\epsilon_{\mathrm{i}} \chi_{\mathrm{i}}$ allows $M$ to be written as

$$
\begin{array}{r}
M=\int \mathrm{d} Q^{\prime} \int \mathrm{d} Q \int \mathrm{d} p\left\langle m_{\mathrm{i}, \mathrm{f}}(P / \mu) \exp \left(i t\left(\epsilon_{\mathrm{i}}\right) / \hbar\right) \chi_{\mathrm{i}} \mid Q^{\prime}\right\rangle \times \\
\left\langle Q^{\prime} \mid p\right\rangle \exp \left(i t\left(E+T_{\text {class }}\right) / \hbar\right)\langle p \mid Q\rangle \times \\
\langle Q| \exp \left(i t\left(V_{\mathrm{f}}(Q) / \hbar\right) m_{\mathrm{i}, \mathrm{f}}(P / \mu) \chi_{\mathrm{i}}\right\rangle
\end{array}
$$

Here $T_{\text {class }}$ is the eigenvalue of the $Q$-coordinate's kinetic energy operator $T$ in the momentum eigenbasis $\{|p\rangle\}, T_{\text {class }}=\left(p^{2} / 2 \mu_{Q}\right)$, and $\left\langle Q^{\prime} \mid p\right\rangle=(2 \pi \hbar)^{-1 / 2} \exp \left(i p Q^{\prime} / \hbar\right)$ is the coordinate representation of the momentum eigenfunction along the active $Q$ coordinate.

The integration over time can be carried out and gives the following expression for the total rate:

$$
\begin{aligned}
R_{\mathrm{T}}= & (2 \pi / \hbar) \int \rho(E) \int \mathrm{d} Q^{\prime} \int \mathrm{d} Q \int \mathrm{d} p\left\langle m_{\mathrm{i}, \mathrm{f}}(P / \mu) \chi_{\mathrm{i}} \mid Q^{\prime}\right\rangle \times \\
& \left\langle Q^{\prime} \mid p\right\rangle \delta\left(T_{\text {class }}+E+V_{\mathrm{f}}(Q)-\epsilon_{\mathrm{i}}\right)\langle p \mid Q\rangle\left\langle Q \mid m_{\mathrm{i}, \mathrm{f}}(P / \mu) \chi_{\mathrm{i}}\right\rangle \mathrm{d} E
\end{aligned}
$$

Since the energy $E$ is restricted to match the state-to-state energy differences $E=\epsilon_{\mathrm{i}}-\epsilon_{\mathrm{f}}$, the integral over $\mathrm{d} E$ in eq 37 can be replaced by a sum over accessible final-state $\epsilon_{\mathrm{f}}$ values multiplied by the spacing between neighboring such states $\left(\mathrm{d} E_{\mathrm{f}}=\epsilon_{\mathrm{f}}-\right.$ $\left.\epsilon_{\mathrm{f}-1}\right)$ :

$$
\begin{array}{r}
R_{\mathrm{T}}=(2 \pi / \hbar) \sum_{\mathrm{f}} \rho\left(\epsilon_{\mathrm{i}}-\epsilon_{\mathrm{f}}\right) \mathrm{d} E_{\mathrm{f}} \int \mathrm{d} Q^{\prime} \int \mathrm{d} Q \int \mathrm{d} p \times \\
\left\langle m_{\mathrm{i}, \mathrm{f}}(P / \mu) \chi_{\mathrm{i}} \mid Q^{\prime}\right\rangle\left\langle Q^{\prime} \mid p\right\rangle \delta\left(T_{\text {class }}+V_{\mathrm{f}}(Q)-\epsilon_{\mathrm{f}}\right)\langle p \mid \mathrm{Q}\rangle \times \\
\left\langle Q \mid m_{\mathrm{i}, \mathrm{f}}(P / \mu) \chi_{\mathrm{i}}\right\rangle
\end{array}
$$

In this form, which is analogous to eq 26 in the photon absorption case, the rate is expressed as a sum over the neutral molecule's vibration-rotation states to which the specific initial state having energy $\epsilon_{\mathrm{i}}$ can decay of (a) a translational state density $\rho$ multiplied by (b) the expectation value of an integral kernel $\Delta$ whose coordinate representation is

$$
\Delta\left(Q^{\prime}, Q\right)=\begin{aligned}
& \int \mathrm{d} p\left|Q^{\prime}\right\rangle m_{\mathrm{i}, \mathrm{f}}\left(Q^{\prime}\right)\left\langle Q^{\prime} \mid p\right\rangle \delta\left(p^{2} / 2 \mu_{Q}+\right. \\
& \left.V_{\mathrm{f}}(Q)-\epsilon_{\mathrm{f}}\right)\langle p \mid Q\rangle m_{\mathrm{i}, \mathrm{f}}(Q)\langle Q|
\end{aligned}
$$

with the expectation value taken for the function $\psi=\left|(P / \mu) \chi_{\mathrm{i}}\right\rangle$ equal to the anion's initial vibration - rotation state acted on by $P / \mu$ (in the harmonic approximation, $P / \mu \chi_{\mathrm{i}}$ would yield a combination of functions of one higher and one lower quantum of vibration or rotation). Another way to view eq 38 is as an integral over $Q$ and $Q^{\prime}$ of $\left\langle m_{\mathrm{i}, \mathrm{f}}(P / \mu) \chi_{\mathrm{i}} \mid Q^{\prime}\right\rangle^{*}\left\langle Q \mid m_{\mathrm{i}, \mathrm{f}}(P / \mu) \chi_{\mathrm{i}}\right\rangle$ multiplied by the (nondiagonal) representation in $Q$ space of the $\delta\left(T+V_{\mathrm{f}}(Q)-\epsilon_{\mathrm{f}}\right)$ operator, which is diagonal in momentum space and equal to $\delta\left(p^{2} / 2 \mu_{Q}+V_{\mathrm{f}}(Q)-\epsilon_{\mathrm{f}}\right)$ but which must be transformed using coordinate space using $\left\langle Q^{\prime} \mid p\right\rangle$ and $\langle p \mid Q\rangle$.

The integral operator $\Delta\left(Q^{\prime}, Q\right)$ can be recast in a different form by carrying out the integration over the $p$ variable in eq
39. Doing so and substituting the result back into the rate expression gives

$$
\begin{gathered}
R_{\mathrm{T}}=(2 \pi / \hbar) \sum_{\mathrm{f}} \rho\left(\epsilon_{\mathrm{i}}-\epsilon_{\mathrm{f}}\right) \mathrm{d} E_{\mathrm{f}} \int \mathrm{d} Q^{\prime} \int \mathrm{d} Q \times \\
\left\langle m_{\mathrm{i}, \mathrm{f}}(P / \mu) \chi_{\mathrm{i}} \mid Q^{\prime}\right\rangle \frac{1}{\pi \hbar} \cos \left\{\left[Q^{\prime}-\right.\right. \\
\left.Q] \sqrt{2 \mu_{Q}\left(\epsilon_{\mathrm{f}}-V_{\mathrm{f}}(Q)\right)} / \hbar\right\} \frac{\mu_{Q}}{\sqrt{2 \mu_{Q}\left(\epsilon_{\mathrm{f}}-V_{\mathrm{f}}(Q)\right)}}\left\langle Q \mid m_{\mathrm{i}, \mathrm{f}}(P / \mu) \chi_{\mathrm{i}}\right\rangle
\end{gathered}
$$

The quantity $\left\{2 \mu_{Q}\left(\epsilon_{\mathrm{f}}-V_{\mathrm{f}}(Q)\right)\right\}^{1 / 2}$ is the classical momentum along the $Q$ coordinate with energy $\epsilon_{\mathrm{f}}$ moving on the neutral molecule's surface $V_{\mathrm{f}}(Q)$, so $\mu_{Q}$ divided into this is the speed of movement at $Q_{0}$ on the neutral molecule's surface.

Bearing in mind the discussion of the nature of the electronic non-BO matrix elements $m_{\mathrm{i}, \mathrm{f}}(Q)$ given in Section I.C, the above rate expression can be further approximated by constraining $Q^{\prime}$ and $Q$ to the region $Q^{\prime}=Q=Q_{0}$ where the anion and neutral surfaces approach most closely

$$
m_{\mathrm{i}, \mathrm{f}}(Q)=\delta\left(Q-Q_{0}\right) m^{*}
$$

where the quantity $m^{*}$ is the integral representing the total strength of the $m_{\mathrm{i}, \mathrm{f}}$ coupling concentrated at the geometry $Q_{0}$ : $m^{*}=\int m_{\mathrm{i}, \mathrm{f}}(Q) \mathrm{d} Q$. Introducing this approximation into eq 40 allows $R_{\mathrm{T}}$ to be written in its simplest form as

$$
\left.R_{\mathrm{T}}=(2 \pi / \hbar) \sum_{\mathrm{f}} \rho\left(\epsilon_{\mathrm{i}}-\epsilon_{\mathrm{f}}\right) \mathrm{d} E_{\mathrm{f}}\left\{(P / \mu) \chi_{\mathrm{i}}\left(Q_{0}\right)\right\}^{2}\right\rangle\left|m^{*}\right|^{2} \frac{1}{\pi \hbar v_{0}}
$$

where $v_{0}$ is the velocity along the $Q$ coordinate at the geometry $Q_{0}$

$$
v_{0}=\frac{2 \mu_{Q}\left(\epsilon_{\mathrm{f}}-V_{\mathrm{f}}\left(Q_{0}\right)\right)}{\mu_{Q}}
$$

and $\left|m_{\mathrm{i}} *\right|^{2}$ is the square of the integrated electronic non-BO matrix element introduced above (n.b., $\left|m^{*}\right|^{2}$ has units of $(\mathrm{g}$ $\left.\mathrm{cm}^{2} / \mathrm{s}\right)^{2}$ ).

The expression shown in eq 42 for the rate of ejection of electrons from a specified initial vibration-rotation state $\chi_{\mathrm{i}}(Q)$ induced by non-BO coupling to all accessible neutral-moleculeplus-free-electron final states (labeled f) gives this rate as:

1. A sum over all final vibration-rotation states $\epsilon_{\mathrm{f}}$ lying below $\epsilon_{\mathrm{i}}$ for which the geometry $Q_{0}$ is within the classically allowed region of the corresponding vibration-rotation wave function $\chi_{\mathrm{f}}(Q)$ (so that $v_{0}$ is real) of

2 . the modulus squared of the function $m^{*}(P / \mu) \chi_{\mathrm{i}}$ evaluated at $Q_{0}$

3. multiplied by the state density $\rho\left(\epsilon_{\mathrm{i}}-\epsilon_{\mathrm{f}}\right) \mathrm{d} E_{\mathrm{f}}$ for the ejected electron and multiplied by $(2 \pi / \hbar)(1 / \pi \hbar)$, and finally

4. divided by the speed $v_{0}$ of passage through $Q_{0}$.

\section{Summary}

The rate of ejection of electrons from anions induced by nonBO couplings can be expressed rigorously and quantum mechanically as a Fourier transform of an overlap function between two functions 


$$
\begin{aligned}
R_{\mathrm{T}}= & (2 \pi / \hbar) \int(1 / 2 \pi \hbar) \int \rho(E)\left\langle m_{\mathrm{i}, \mathrm{f}}(P / \mu) \exp (i t(T+\right. \\
& \left.\left.V_{\mathrm{i}}\right) / \hbar\right) \chi_{\mathrm{i}}\left|\exp \left(i t\left(E+T+V_{\mathrm{f}}\right) / \hbar\right) m_{\mathrm{i}, \mathrm{f}}(P / \mu) \chi_{\mathrm{i}}\right\rangle \mathrm{d} t \mathrm{~d} E
\end{aligned}
$$

one of which is the initial vibration-rotation function $\chi_{\mathrm{i}}$ acted on by the non-BO perturbation $m_{\mathrm{i}, \mathrm{f}}(P / \mu)$ and then propagated on the neutral molecule surface, the other being the initial $\chi_{\mathrm{i}}$ propagated on the anion surface and then acted on by $m_{\mathrm{i}, \mathrm{f}}(P / \mu)$. In computer applications, it would be efficient to compute $R_{\mathrm{T}}$ in this manner only if long-time surface propagation tools are applicable.

By introducing the simplest semiclassical approximation to the propagators, in which the nucler motion kinetic energy is assumed to commute with the anion and neutral potential energy functions and with the non-BO coupling operators, one obtains

$$
R_{\mathrm{T}}=(2 \pi / \hbar) \int \rho(E)\left\langle m_{\mathrm{i}, \mathrm{f}}(P / \mu) \chi_{\mathrm{i}}\left|\delta\left(V_{\mathrm{f}}+E-V_{\mathrm{i}}\right)\right| m_{\mathrm{i}, \mathrm{f}}\right.
$$$$
(P / \mu) \chi_{\mathrm{i}}>\mathrm{d} E
$$

Unlike its success in treating the photon absorption rate expression, this simplest approximation produces a nonsensical expression in the present case because there are no geometries at which $\left(V_{\mathrm{f}}+E-V_{\mathrm{i}}\right)=0$, as a result of which $R_{\mathrm{T}}$ is predicted to vanish. In the photon absorption situation, there are geometries at which the classical nuclear-motion momentum is conserved (i.e., where the excited and shifted ground-state surfaces intersect). In the non-BO transition case, such geometries do not exist because the transition is not one in which the nuclear-motion momentum is conserved. Quite to the contrary, non-BO transitions involve the simultaneous interchange of energy (from the nuclei to the electrons) and of momentum and/or angular momentum.

Improving on the treatment of the vibration-rotation motion only slightly allows $R_{\mathrm{T}}$ to be recast in the so-called semiquantum form

$$
\begin{aligned}
& R_{\mathrm{T}}= \\
& (2 \pi / \hbar) \sum_{\mathrm{f}} \rho\left(\epsilon_{\mathrm{i}}-\epsilon_{\mathrm{f}}\right) \mathrm{d} E_{\mathrm{f}} \int \mathrm{d} Q^{\prime} \int \mathrm{d} Q\left\langle m_{\mathrm{i}, \mathrm{f}}(P / \mu) \chi_{\mathrm{i}} \mid Q^{\prime}\right\rangle \frac{1}{\pi \hbar} \times \\
& \cos \left\{\left[Q^{\prime}-Q\right] \sqrt{\left.2 \mu_{Q}\left(\epsilon_{\mathrm{f}}-V_{\mathrm{f}}(Q)\right) / \hbar\right\}} \frac{\mu_{Q}}{\sqrt{2 \mu_{Q}\left(\epsilon_{\mathrm{f}}-V_{\mathrm{f}}(Q)\right)}}\right. \\
& \left\langle Q \mid m_{\mathrm{i}, \mathrm{f}}(P / \mu) \chi_{\mathrm{i}}\right\rangle
\end{aligned}
$$

that may prove computationally useful in cases where the geometry $(Q)$ dependence of the non-BO electronic matrix element $m_{\mathrm{i}, \mathrm{f}}$ is known. In this expression, the rate is given in terms of the functions $m_{\mathrm{i}, \mathrm{f}}(P / \mu) \chi_{\mathrm{i}}$, the density of state function $\rho$, the classical momentum on the neutral molecule's surface, and a cos function whose argument is the classical action connecting the points $Q^{\prime}$ and $Q$ via the momentum on the neutral molecule's surface with an energy $\epsilon_{\mathrm{f}}$. This cos function will oscillate rapidly when this action is large, so its dominant contributions to the rate arise for small momenta and/or small $Q^{\prime}-Q$ values.

Finally, by using what is known about the geometry dependence of the $m_{\mathrm{i}, \mathrm{f}}$ functions (i.e., that $m_{\mathrm{i}, \mathrm{f}}$ is strongly "peaked" near geometries $Q_{0}$ where the anion and neutral surfaces approach most closely), it is possible to further simplify the semiquantum equation for $R_{\mathrm{T}}$

$$
\left.R_{\mathrm{T}}=(2 \pi / \hbar) \sum_{\mathrm{f}} \rho\left(\epsilon_{\mathrm{i}}-\epsilon_{\mathrm{f}}\right) \mathrm{d} E_{\mathrm{f}}\left\{(P / \mu) \chi_{\mathrm{i}}\left(Q_{0}\right)\right\}^{2}\right\rangle\left|m^{*}\right|^{2} \frac{1}{\pi \hbar v_{0}}
$$

to one that requires knowledge of the derivative of the initialstate vibrational wave function $\left(P / \mu \chi_{\mathrm{i}}\right)$ evaluated at $Q_{0}$, the speed $v_{0}$ at which classical motion on the neutral molecule surface passes through $Q_{0}$, the density of states $\rho$, and the magnitude of the integrated strerength $m^{*}$ of $m_{\mathrm{i}, \mathrm{f}}$ at $Q_{0}$.

It should be emphasized that it is not the modulus $\left|\chi_{\mathrm{i}}\left(Q_{0}\right)\right|^{2}$ that enters into the weighting functiion in eqs 41 or 42 ; it is the derivative $\left(P / \mu \chi_{\mathrm{i}}\right)$ whose modulus squared enters. In contrast, in the photon absorption case, the rate involves, as given in eq 21

$$
\begin{array}{r}
R_{\mathrm{T}}=(2 \pi / \hbar)(1 / 2 \pi \hbar) \int \exp [-i t \omega]\left\langle\exp \left(i t h_{\mathrm{i}} / \hbar\right) \chi_{\mathrm{i}}\right| \mu_{\mathrm{i}, \mathrm{f}} * \times \\
\exp \left(i t h_{\mathrm{f}} / \hbar\right) \mu_{\mathrm{i}, \mathrm{f}}\left|\chi_{\mathrm{i}}\right\rangle \mathrm{d} t
\end{array}
$$

the modulus squared of $\chi_{i}$ itself. The qualitative difference in the two cases has to do with the inherent requirement that the nuclear-motion momentum and/or angular momentum change in non-BO transitions while the same quantities are preserved in photon absorption events (in the semiclassical treatment).

In closing, it should also be mentioned that significant extension of the developments given here, including the relation to the Landau-Zener theory of surface hopping, will appear in Advances in Quantum Chemistry ${ }^{11}$ in the near future.

Acknowledgment. This work has been supported by NSF Grant CHE-9618904 and by proceeds of the Henry Eyring Endowed Chair.

\section{References and Notes}

(1) (a) Lykke, K. R.; Neumark, D. M.; Ansersen, T.; Trapa, V. J.; Lineberger, W. C. J. Chem. Phys. 1987, 87, 6842. (b) Neumark, D. M.; Lykke, K. R.; Andersen, T.; Lineberger, W. C. J. Chem. Phys. 1985, 83, 4364. (c) Andersen, T.; Lykke, K. R.; Neumark, D. M.; Lineberger, W. C. J. Chem. Phys. 1987, 86, 1858

(2) (a) Marks, J.; Wentzel, D. M.; Comita, P. B.; Brauman, J. I. J. Chem. Phys. 1986, 86, 5284. (b) Meyer, F. K.; Jasinski, J. M.; Rosenfeld, R. N.; Brauman, J. I. J. Am. Chem. Soc. 1982, 104, 663. (c) Rosenfeld, R. N.; Jasinski, J. M.; Brauman, J. I. J. Chem. Phys. 1979, 71, 1030. Mead, R. D.; Lykke, K. R.; Lineberger, W. C.; Marks, J.; Brauman, J. I. J. Chem. Phys. 1984, 81, 4883. (d) Jackson, R. L.; Zimmerman, A. H.; Brauman, J. I. J. Chem. Phys. 1979, 71, 2088. (e) Zimmerman, A. H.; Reed, K. J.; Brauman, J. I. J. Am. Chem. Soc. 1977, 99, 7203. (f) Zimmerman, A. H.; Brauman, J. I. J. Chem. Phys. 1977, 66, 5823.

(3) Wight, C. A.; Beauchamp, J. L. J. Am. Chem. Soc. 1981, 103, 6501

(4) (a) Acharya, P. K.; Kendall, R.; Simons, J. Vibration-Induced Electron Ejection in Molecular Anions. Contrib.-Symp. At. Surf. Phys., 1984 1984. (b) Acharya, P. K.; Kendall, Rick A.; Simons, J. J. Am. Chem. Soc. 1984, 106, 3402. (c) Acharya, P. K.; Kendall, R.; Simons, J. J. Chem. Phys. 1985, 83, 3888. (d) Chalasinski, G.; Kendall, R. A.; Taylor, H.; Simons, J. J. Phys. Chem. 1988, 92, 3086. (e) O’Neal, D.; Simons, J. J. Phys. Chem. 1988, 93, 58. (f) Simons, J. J. Chem. Phys. 1989, 91, 6858. (g) Simons, J. J. Am. Chem. Soc. 1981, 103, 3971.

(5) Clary, D. L. J. Phys. Chem. 1988, 92, 3173.

(6) Berry, R. S. J. Chem. Phys. 1966, 45, 1228

(7) (a) Simons, J. J. Phys. Chem. 1982, 86, 3615. (b) Taylor, H.; Simons, J. J. Phys. Chem. 1986, 90, 580. (c) Lax, M. J. Chem. Phys. 1952 , 30, 1752. (d) Noda, C.; Zare, R. N. J. Mol. Spectrosc. 1982, 95, 254.

(8) (a) A good textbook treatment of how time correlation functions relate to photon absorption and other rates is given in: McQuarrie, D. A. Statistical Mechanics; Harper and Row: New York, 1976. (b) Gordon, R. G. Adv. Mag. Reson. 1968, 3, 1 .

(9) (a) Bergsma, J. P.; Berens, P. H.; Wilson, K. R.; Fredkin, D. R.; Heller, E. J. J. Phys. Chem. 1984, 88, 612. (b) Heller, E. J.; Brown, R. C. J. Chem. Phys. 1983, 79, 3336. (c) Heller, E. J. J. Chem. Phys. 1978, 68 2066. (d) Heller, E. J. J. Chem. Phys. 1978, 68, 3891.

(10) (a) Landau, L. Phys. Z. Sowjetunion 1932, 1, 88. (b) Zener, C. Proc. R. Soc. London 1932, A137, 696.

(11) Simons, J. Adv. Quantum Chem., to be published. 